Relations industrielles

Industrial Relations

\title{
Managing Human Resources, par Leonard R. Sayles et George Strauss Prentice-Hall Inc., Englewood Cliffs, N.J., 1977, 528 pp.
}

\section{Laurent Bélanger}

Volume 32, numéro 1, 1977

URI : https://id.erudit.org/iderudit/028775ar

DOI : https://doi.org/10.7202/028775ar

Aller au sommaire du numéro

Éditeur(s)

Département des relations industrielles de l'Université Laval

ISSN

0034-379X (imprimé)

1703-8138 (numérique)

Découvrir la revue

Citer ce compte rendu

Bélanger, L. (1977). Compte rendu de [Managing Human Resources, par Leonard R. Sayles et George Strauss Prentice-Hall Inc., Englewood Cliffs, N.J., 1977, 528 pp.] Relations industrielles / Industrial Relations, 32(1), 147-147.

https://doi.org/10.7202/028775ar

Tous droits réservés @ C Département des relations industrielles de l'Universite Laval, 1977
Ce document est protégé par la loi sur le droit d'auteur. L'utilisation des services d'Érudit (y compris la reproduction) est assujettie à sa politique d'utilisation que vous pouvez consulter en ligne.

https://apropos.erudit.org/fr/usagers/politique-dutilisation/ 
gique certaine. Citons à titre d'exemples les contributions de MM. François Perroux, «Activité économique et science économique» (pp. 14-74), Claude Rivière, «Le changement social», (pp. 316-337) et Émile Poulat, «Sociologie religieuse» (pp. 399414). Signalons aussi deux articles sur les organisations (Erhard Freidberg, pp. 346365 ) et sur la sociologie du travail (Roland Guillon, pp. 509-532) qui peuvent constituer des introductions intéressantes à ces thèmes.

Il ne faut pas s'attendre à trouver dans cet ouvrage «le bilan le plus actuel qui soit des recherches en cours " en sciences sociales qui nous promet la jaquette du livre; on y présente des synthèses honnêtes qui satisferont le profane qui apprendront peu à celui qui est le moindrement familier avec les sujets abordés.

Gilles DUSSAULT

Université laval

Managing Human Resources, par Leonard R. Sayles et George Strauss PrenticeHall Inc., Englewood Cliffs N. J., 1977, $528 \mathrm{pp}$.

Cet ouvrage est un réaménagement et une mise-à-jour d'un volume précédent qui a connu plusieurs éditions et une large diffusion surtout aux États-Unis et au Canada, tellement qu'il n'est pas utile ici de faire une longue présentation. On y retrouve évidemment toute les rubriques qu'on utilise habituellement pour décrire le domaine de la gestion des ressources humaines dans une entreprise syndiquée Quelques chapitres ont été ajoutés pour tenir compte du développement des sciences du comportement et de la législation nouvelle à l'endroit des groupes minoritaires et de l'emploi de la main-d'œuvre féminine. L'ouvrage se distingue de d'autres dans le même domaine par un traitement du sujet en des termes très simples et qui collent de près à la pratique quotidienne des relations industrielles. Les auteurs s'en tiennent à une vision uniquement descriptive de la place d'un service de personnel dans une entreprise, des objectifs poursuivis, et des activités qui y sont accomplies. Pour s'en rendre compte, il suffit de s'arrêter aux nombreux exemples fortement particularisés et insérés dans le texte, ou encore aux incidents qui apparaissent à la fin de la plupart des chapitres.

Ce volume serait surtout utile à ceux qui veulent se donner une formation initiale à la gestion des ressources humaines ou à ceux qui débutent dans cette carrière sans cette formation initiale adéquate. Il serait également souhaitable qu'on en fasse une traduction en français.

Laurent BÉLANGER

Université Laval

Health Economics Symposium: Proceedings of the First Canadian Conference, by R. D. Fraser, Kingston, Industrial Relations Center, Queen's University, 1976, $209 \mathrm{pp}$.

Cet ouvrage rend compte de la première conférence canadienne sur l'économique de la santé, tenue en septembre 1974 à l'Université Queen's. On a voulu, pour cette première, faire un inventaire des questions, reliées à la santé, présentant un intérêt pour l'économiste; cependant c'est le thème de la rémunération des médecins qui revient le plus souvent dans les propos des conférenciers et qui tend à apparaître comme central (R. G. Evans, "Modeling the Economic Objectives of the Physician», pp. 33-46; T. J. Boudreau et J. Y. Rivard, «An Evaluation of Different Methods for Remunerating Physicians», pp. 52-62; P. Ruderman, «The Political Economy of Fee-Setting and the Future of Fee-forService», pp. 75-90 et R. G. Beck, «Some Dynamic Effects of Copayment on Utilization of Medical Services in Saskatchewan », pp. 98-110). Les auteurs s'interrogent sur le paiement à l'acte et ses alternatives - capitation, salariat, vacation, formules mixtes - comme modes de rémunération des professionnels de la santé; ils essaient particulièrement de mesurer leur impact respectif sur les coûts des services ainsi que sur les types de comportements économiques qu'ils engendrent (profils de pratique). De fait, on est loin de l'unanimité quant à la valeur des différentes façons de payer les médecins, mais tous s'entendent pour dire que le choix d'un mode de paiement des services médicaux est plutôt d'ordre politique qu'économique.

L'éditeur, dans sa présentation des textes des conférences, propose au lecteur une introduction générale, fort intéressante, aux dimensions économiques des soins de santé (K. Adams et R. D. Fraser, «An Overview of the Canadian Health Care System», pp. 6-32). Les données qu'h présente illustrent bien l'importance économique qu'a prise la santé depuis une vingtaine d'années au Canada. Par exemple, les dépenses en soins personnels sont passées de 1.5 milliards de dollars à 5.1 milliards de 1960 à 1971, soit une augmentation de 